\title{
First Results from the HERMES Recoil Detector
}

\author{
A. Mussgiller* and D. Zeiler \\ (On behalf of the HERMES collaboration) \\ University of Erlangen-Nuremberg, Erlangen, Germany \\ E-mail: andreas.mussgiller@desy.de
}

\begin{abstract}
For the final running period of HERA, a Recoil Detector was installed at the HERMES experiment to improve measurements of hard exclusive processes. Here, Deeply-Virtual Compton scattering is of particular interest, as this process provides constraints on Generalized Parton Distributions that give access to the total angular momenta of quarks inside the nucleon.

The HERMES Recoil Detector was designed to improve the selection of exclusive events by a direct measurement of the four-momentum of the recoiling particles. It consisted of three components: two layers of double-sided silicon sensors inside the HERA vacuum, a two-barrel scintillating fiber tracker, and a photon detector. All sub-detectors were located inside a 1 Tesla solenoidal magnetic field.

The Recoil Detector was installed in late 2005. After the commissioning of all components was finished in September 2006, it was working stable until the end of data taking at HERA on June 30 of 2007. Results on the performance and calibration of the silicon detector are presented.
\end{abstract}

Vertex 2008 - 17th International Workshop on Vertex detectors

July 28 - August 1, 2008

Utö Island, Sweden

\footnotetext{
* Speaker.
} 


\section{Contents}

1. Introduction 2

2. The HERMES Experiment 3

3. The Recoil Detector 3

4. The Recoil Silicon Detector 4

4.1 Raw Data Corrections 4

4.1.1 Pedestal Stability 5

4.1.2 Correlated Noise 5

$\begin{array}{ll}4.1 .3 \text { Crosstalk } & 7\end{array}$

4.2 Calibration 8

5. Summary and Outlook 9

\section{Introduction}

Recently there has been an increasing interest in the formalism of Generalized Parton Distributions (GPDs) as they allow a consistent description of various aspects of nucleon structure. Certain limits and moments of the GPDs incorporate the well-known nucleon form factors determined from elastic scattering as well as parton distributions functions (PDFs) determined from measurements of inclusive and semi-inclusive deep inelastic lepton-nucleon scattering (DIS and SIDIS, respectively). Strong interest in the GPD framework has evolved because of the fact that GPDs encode the unknown total angular momenta of quarks and gluons within the nucleon $\left(J_{q}\right.$ and $J_{g}$, respectively) [1]. The orbital angular momenta of quarks $\left(L_{q}\right)$ can be calculated by knowing the GPDs and the quarks' contribution to the spin of the nucleon.

The theoretically cleanest process known to constrain GPDs is Deeply-Virtual Compton Scattering (DVCS), in which a highly virtual photon (emitted by the incoming beam lepton) is absorbed by a parton of the target nucleon and produces a single real photon in the final soutate along with the recoiling nucleon in its ground state.

The DVCS process has the same final state as the Bethe-Heitler $(\mathrm{BH})$ process, in which a real photon is radiated by either the incident or the scattered lepton. At HERMES kinematics the $\mathrm{BH}$ process is the dominant contribution to the cross section. However, the DVCS amplitude can be accessed via the interference term by measuring various cross section asymmetries and their dependence on the azimuthal angle $\phi$, which is defined as the angle between the lepton scattering plane and the photon production plane. 


\section{The HERMES Experiment}

HERMES [2] was a fixed target experiment that used the $27.6 \mathrm{GeV}$ electron and positron beam provided by HERA at DESY in Hamburg. For the data collected up to 2005, exclusive DVCS events were selected by identifying the scattered beam lepton and the produced real photon in the HERMES spectrometer. The recoiling proton remained undetected and therefore a cut on the missing mass was applied. For the last two data taking years (2006 and 2007) a new detection system was installed in the target region in order to detect the recoiling proton from exclusive reactions and hence to improve the selection of those events.

\section{The Recoil Detector}

The HERMES Recoil Detector consisted of three sub-detector components (see figure 1). The innermost detector was a silicon detector (SSD) made up of 16 double-sided silicon strip detectors arranged in two layers around the target cell inside the HERA beam vaccum. It will be described in detail in the following sections. The second component was a scintillating fiber tracker (SFT) made up of two barrels each with two layers of fibers parallel to the beam and two layers of fibers with a $10^{\circ}$ stereo angle. The outermost detector component was a photon detector (PD) consisting of 3

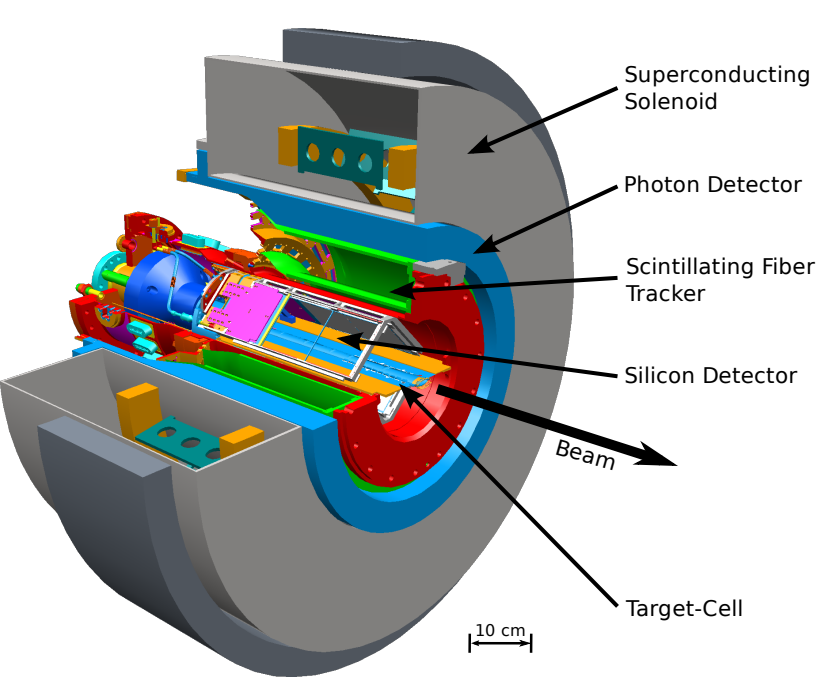

Figure 1: A 3D CAD drawing of the Recoil Detector. layers each with a tungsten and a scintillator sub-layer. The whole detection system was contained inside the 1 Tesla field of a superconducting solenoid. Both the SSD and the SFT provide energy and position information which is used in the track and momentum reconstruction. The left panel of figure 2 shows a schematic view of the Recoil Detector in beam direction. The right plot shows the energy deposit in the inner silicon layer vs. the energy deposit in the outer silicon layer with the characteristic bands for protons and deuterons. Starting from a momentum of about $125 \mathrm{MeV} / \mathrm{c}$ protons punched through the inner SSD layer and got stopped in the outer SSD layer as indicated by the left track in figure 2 (blue). In this case the sum of the two SSD energy deposits corresponds to the kinetic energy of the particle before it entered the inner SSD layer. With knowledge of the particles identity, obtained from the characteristic energy deposits in both layers (see proton and deuteron bands in figure 2), the momentum can be calculated. At $p \approx 145 \mathrm{MeV} / \mathrm{c}$ protons also punched through the outer SSD layer, but did not have enough energy to reach the SFT layers (indicated by the middle track in the figure; magenta). Here the momentum is reconstructed by both SSD energy deposits and the $d E / d x$ behaviour for protons in silicon. For even higher momenta $(p>200 \mathrm{MeV} / \mathrm{c})$ the additional position 
information from the SFT layers is used to reconstruct the momentum via the bending in the magnetic field (indicated by the right track in the figure; green). In all three cases the energy deposits in the passive materials along the track are taken into account in the momentum reconstruction. For tracks reconstructed via their bending in the magnetic field, the energy information from both SSD and SFT is used for particle identification (PID). The information from the PD is not directly used in the track reconstruction but available for PID at a later stage.
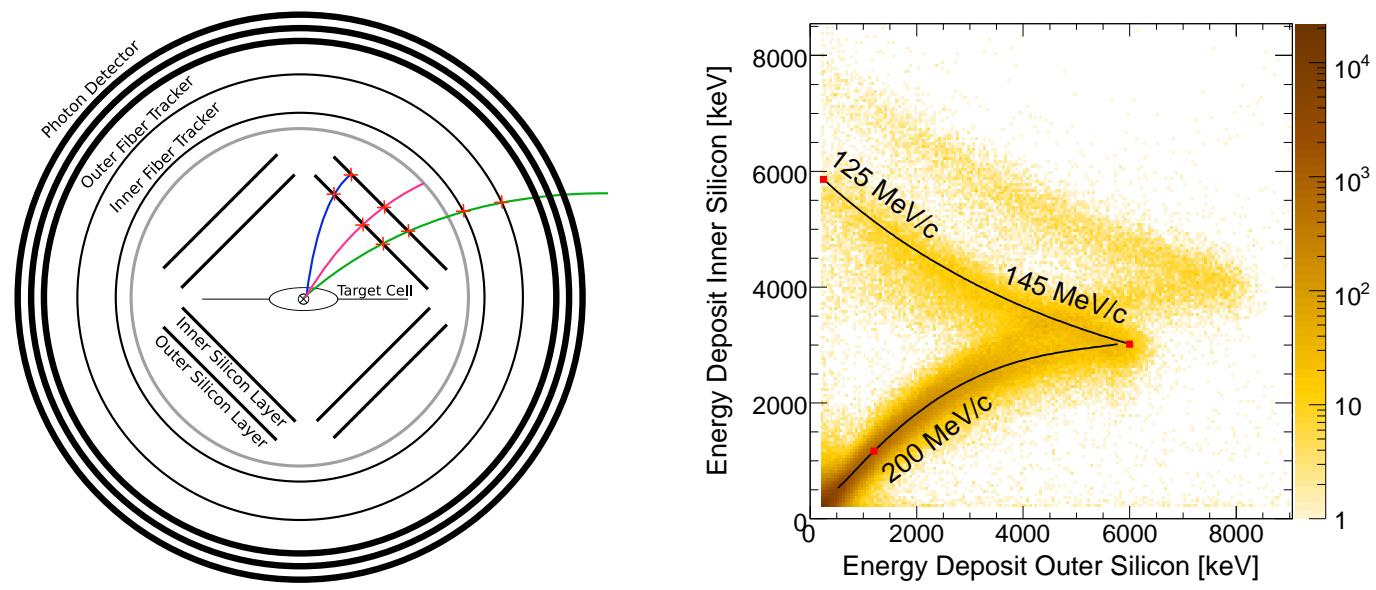

Figure 2: Left: Schematic view of the Recoil Detector in beam direction with three tracks showing the various track reconstruction momentum regions. Right: Energy deposit in the inner SSD layer vs. energy deposit in the outer SSD layer. The black line represent the results from a Geant 4 simulation using protons. The second band corresponds to deuterons stopped in the outer SSD layer.

\section{The Recoil Silicon Detector}

All 16 sensors had an active area of $9.9 \times 9.9 \mathrm{~cm}^{2}$, a pitch of $758.2 \mu \mathrm{m}$ and a thickness of $300 \mu \mathrm{m}$ (N-type bulk material; TTT design by Micron Semiconductors). Two sensors were glued into a ceramics holding frame and combined with the front-end read-out electronics to form a module. Figure 3 shows a sketch of the front and back side of one module. The strips on the p-side (n-side) of each sensor were oriented parallel (perpendicular) to the HERA lepton beam axis. The signals from the 128 strips on each $\mathrm{p}$ - and $\mathrm{n}$-side are routed to the front-end hybrid via Kapton flex-foils. On the hybrid the signals are split in a charge divider network with a ratio of $1: 5$ into a high- (HG) and a low-gain (LG) component to increase the dynamic range. For each sensor side the HG and LG signals are fed to two Helix 3.0 front-end amplifier chips [3].

A total of eight modules were mounted on an aluminum holding structure to build the Recoil Silicon detector. To minimize the amount of passive material (Kapton flex-foils) between the inner and outer SSD layer, the inner and outer modules were mounted such that the p-sides faced each other.

\subsection{Raw Data Corrections}

For very low energy protons the momentum is reconstructed by the energy deposits in both SSD layers. A precise energy measurement is therefore essential for a good performace of the 


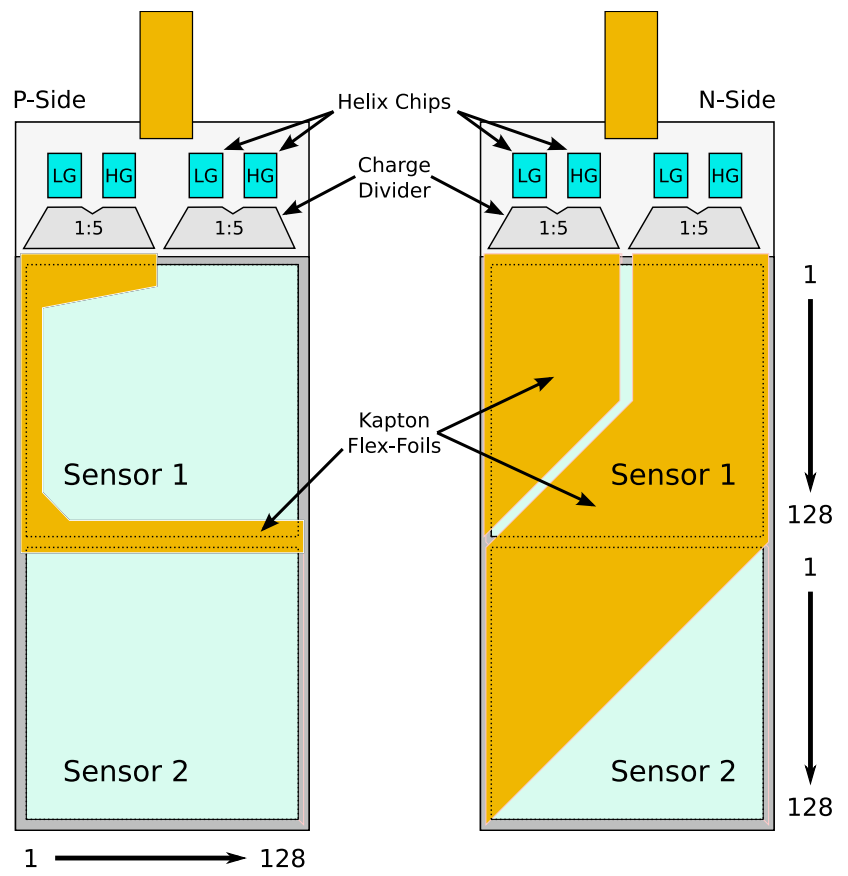

Figure 3: Sketch of the front- and backside of one module of the Recoil Silicon detector.

reconstruction. To achive this, several correction on the raw data must be applied which will be described in the following.

\subsubsection{Pedestal Stability}

During data taking every 2 - 3 hours a dedicated pedestal run was taken. The left plot of figure 4 shows the measured pedestal positions for three channels for about two month worth of pedestal data. The channels indicated by the green (red) data points show large (medium) fluctuations, whereas the channel indicated by the blue data points only shows a slow drift with time. In the right plot of figure 4 the same pedestal data are dispayed vs. the beam current at which the pedestal run was taken, using the pedestal position without beam as an absolute reference. For the channel indicated by the green and red data points a clear dependence of the pedestal position on the beam current is visible.

For both the high and the low gain channel of each strip a parameterization of the beam current dependence is used to correct for the drift of the pedestal between two pedestal runs. For each event and channel the parameterizations give an offset based on the beam current change between the last pedestal run and the current event. This offset is added to the raw data to correct for the drift.

\subsubsection{Correlated Noise}

The ADC used to digitize the data coming from the Helix chips performed an online commonmode correction based on the data from the first 16 channels of each chip. For pure common-mode noise this implementation is sufficient. However, the data contain additional correlated noise which cannot be corrected for by this common-mode correction algorithm. 

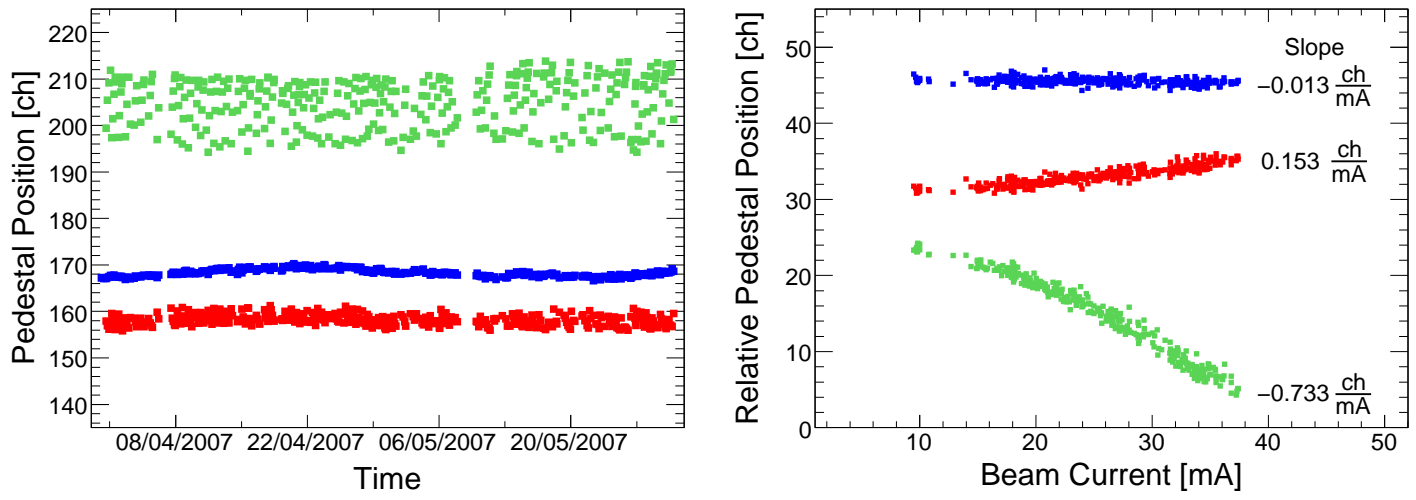

Figure 4: Left: Measured pedestal position for three sample channels vs. time for about two month of pedestal data. Right: The same pedestal data displayed vs. the beam current at which the data was taken. The pedestal data without beam is used as an absolute reference.
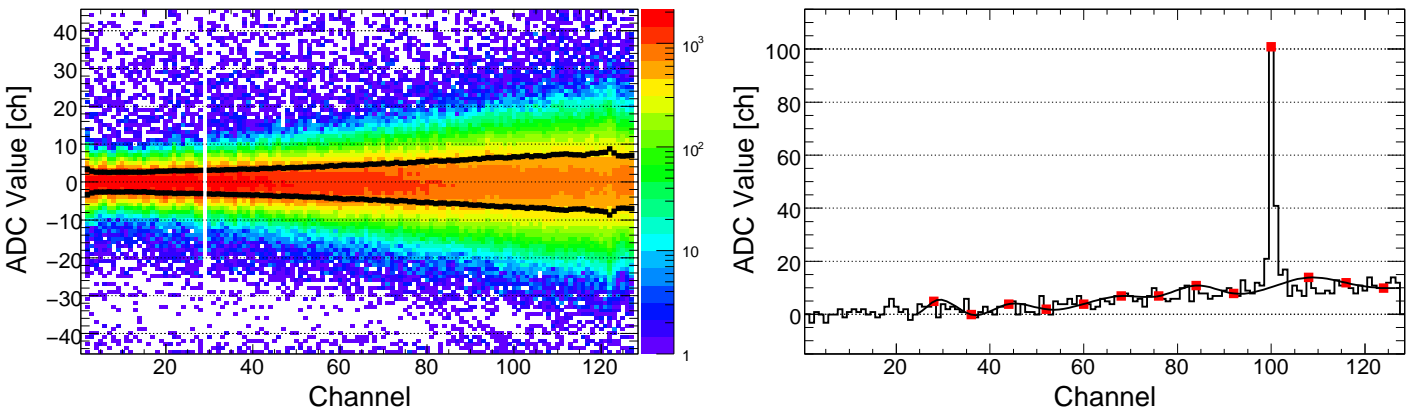

Figure 5: Left: Pedestal for all channels of a chip vs. channel number after pedestal subtraction and common-mode correction in the ADC. Right: Data after pedestal subtraction and common-mode correction for a single event. The red markers indicate the channels used for the correlated noise correction. The data was taken without zero-suppression.

The left plot of figure 5 shows the ADC value for the 128 channels of a chip after pedestal subtraction and common-mode correction performed by the ADC. The black lines in the figure indicate the $\pm 1 \sigma$ band of the pedestal width. The common-mode correction performs well in the beginning of a chip (up to $\approx$ channel 20). Beyond this region, the width of the pedestal increases with increasing channel number. In the right plot of figure 5 the ADC values of all channels of a chip vs. channel number are shown for a single event. At around channel 100 one can observe an actual hit with an amplitude of 100 ADC counts which is, however, incorrect as the baseline for this event drifts towards positive ADC values for increasing channel numbers.

To correct for this drift the data from every $8^{\text {th }}$ channel were always read out and used as basepoints for a spline interpolation. In the figure the basepoints are indicated by the read markers. The actual spline is shown as a line through the basepoints. The result from the interpolation is used to correct for the drift. In case a spline interpolation base point is part of an actual cluster, its signal is replaced by the average signal of both neighbouring base points. 

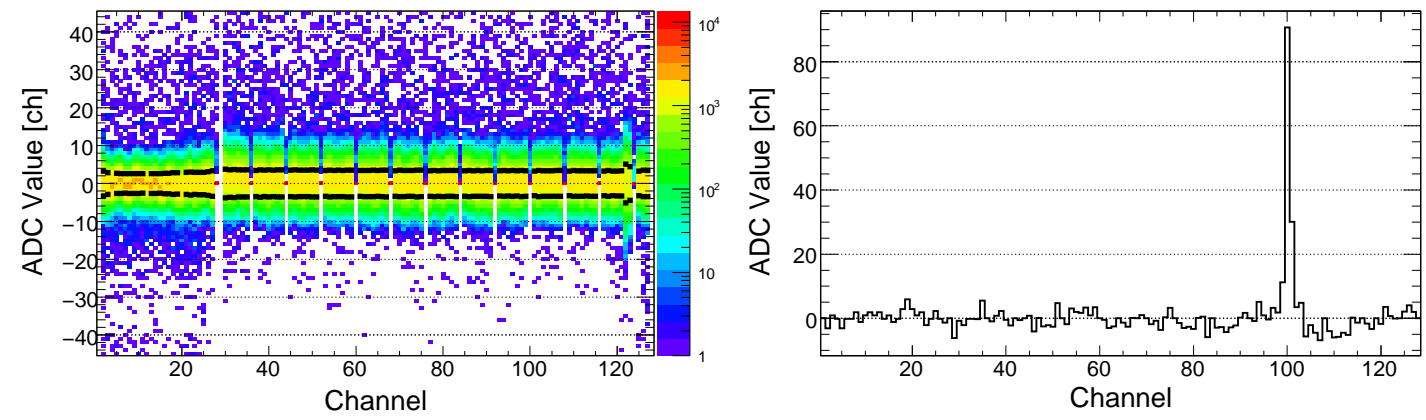

Figure 6: Left: Pedestal for all channels of a chip vs. channel number after the correction for the correlated noise. Right: Data for the sample event from the previous figure after correction for the correlated noise.

In the right plot of figure 6 the same event is shown after the spline-interpolation-correction. The baseline over the full chip is flat and the amplitude of the hit at channel 100 is reduced to approximately 90 ADC counts. For a larger data sample the spline interpolation corrected ADC values vs. channel number are shown in the left plot of the figure. The black lines again indicate the $\pm 1 \sigma$ band of the pedestal width. After the correction the pedestal width is below 3 to 4 ADC counts over the full chip.


Figure 7: ADC value of the channel to the left/right of the channel with the highest ADC value in a cluster (central channel) vs. the ADC value of the central channel before crosstalk correction.

\subsubsection{Crosstalk}

For each actual signal in a channel a significant fraction of that signal migrates into the neighbouring channels. This crosstalk is due to coupling on the flex foils, and the chip itself. Figure 7 shows the ADC value of the neighbouring channels vs. the ADC value of the central channel of a cluster, were central channel denotes the channels with the highest ADC value. Both to the left and to the right a band is seen. The slopes of the bands correspond to the constant fraction of signal that migrates to the neighbours. The crosstalk to the left neighbour is in the order of 11 to $16 \%$ whereas to the right it is in the order of 15 to $21 \%$. In addition, the crosstalk is different for even and odd numbered strips.

The large left-right asymmetry in the crosstalk would introduce a bias in the position measurement in the silicon detector and therefore needs to be corrected for. The algorithm assumes that the 

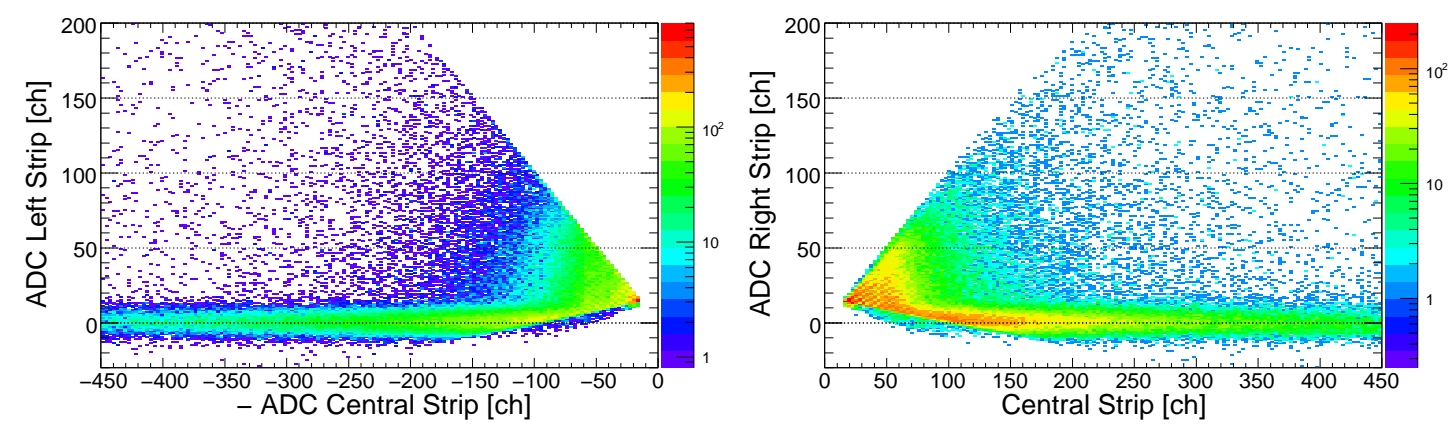

Figure 8: ADC value of the channel to the left/right of the channel with the highest ADC value in a cluster (central channel) vs. the ADC value of the central channel after crosstalk correction.

128 ADC values before correction originate from a $128 \times 128$ matrix times the 128 actual ADC values. The matrix is filled with the fractions of signal remaining in the central channel (diagonal elements) and migrating to the neighbouring channels (off diagonal elements). The crosstalk to the second neighbour is in the order of $2 \%$. Crosstalk effects to channels farther away from the central channel are insignificant and therefore neglected in the correction. By matrix inversion ${ }^{1}$ it is possible to reconstruct the actual ADC values. Figure 8 shows the ADC values of the left and the right neighbour vs. the ADC value in the central channel after the crosstalk correction. After correction the bands are horizontal.

\subsection{Calibration}

For protons stopped in the outer SSD layer as well as for protons with too low momentum to reach the SFT layers, the momentum is reconstructed by just the energy deposits in both SSD layers. In this momentum region the quality of the momentum reconstruction is therefore directly related to the precision of the energy calibration. In a first iteration the calibration is obtained via the energy deposits for protons and deuterons stopped in the outer SSD layer and protons that punch through this layer.

Figure 9 shows the energy deposit in the inner silicon layer vs. the energy deposit in the outer SSD layer for the Hydrogen (left plot) and the Deuterium (right plot) target after a correction for the incident angle. The black curves in the plots show the results of a Geant 4 simulation with protons and deuterons penetrating the sensors under an angle of $90^{\circ}$. The red markers show the position of the stopped proton and deuteron bands (right plot), and the band for protons that punch through the outer SSD layer (left plot), as provided by fits to data. The final calibration is obtained by minimizing the difference between the measured band positions (red markers) and the simulated curves. The behaviour of the system is assumed to be linear, therefore the energy deposits in the inner and outer layers can be expressed by

$$
d E_{i}=c_{i} \cdot A D C_{i}^{\text {cluster }} \text { and } \quad d E_{o}=c_{o} \cdot A D C_{o}^{\text {cluster }}
$$

\footnotetext{
${ }^{1}$ The correction algorithm assumes that crosstalk affects only the immediate and second neighbours to the left and right of the central channel. It is therefore enough to invert a $5 \times 5$ matrix to perform the correction.
} 

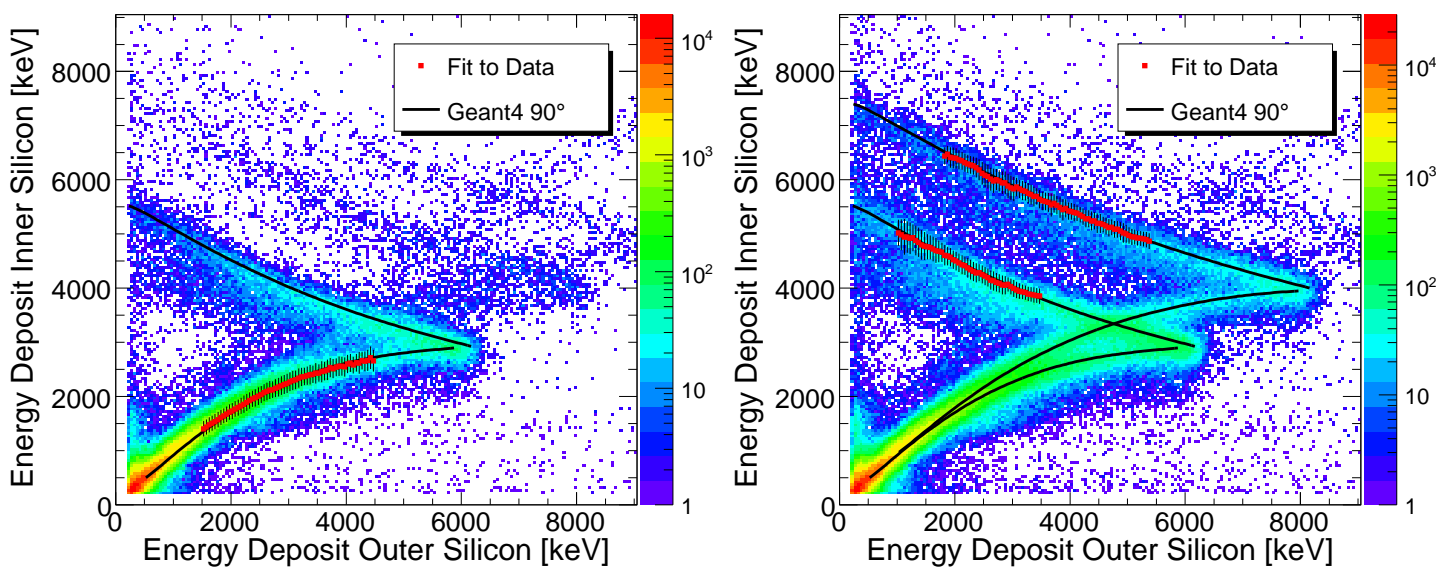

Figure 9: Energy deposit in the inner SSD layer vs. energy deposit in the outer SSD layer for the Hydrogen (left plot) and the Deuterium (right plot) target. The black curves represent the result from a Geant 4 simulation. The red markers indicate the position of the stopped proton and deuteron bands (right plot) and the band for protons that punch through the outer layer (left plot).

were $A D C_{i / o}^{\text {cluster }}$ are the sums of ADC values in a cluster, $d E_{i / o}$ are the calibrated energy deposits and $c_{i / o}$ are the calibration coefficients which are the only two free parameters in the minimization.

\section{Summary and Outlook}

The Recoil Detector was installed at the HERMES experiment in the beginning of 2006. After some set backs during the commissioning phase all three detector components were ready for operation in September of 2006. Until the end of data taking at HERA the Recoil Detector was operating stable and recorded valueable data. Over the nine month of operation, the data taking efficiency of the silicon detector was above $95 \%$.

All raw data correction algorithms have been implemented in the data processing chain and show satisfying results. A first version of the SSD calibration is available, which is used to perform first physics studies in the direction of DVCS and other exclusive channels. However, this calibration neglects any non-linearity of the Helix chips and electronics and is based on large energy deposits $(d E>1.5 \mathrm{MeV}$, see left plot of figure 9). Although for lower energy deposits in the SSD and therefore higher proton momenta, the momentum is reconstructed by the bending in the magnetic field, including energy deposit information will lead to a substantially better precision of the reconstruction. It is therefore planned to improve the calibration in a next iteration and include potential non-linearities and cover the full range of energy deposits.

\section{References}

[1] X. Ji, Phys. Rev. Lett. 78 610, (1997); Phys. Rev. D55 7114, (1997).

[2] HERMES Coll., K. Ackerstaff et al., Nucl. Instr. and Meth. A417 230, (1998).

[3] W. Fallot-Burghardt, A CMOS Mixed-Signal Readout Chip for the Microstrip Detectors of HERA-B, $\mathrm{PhD}$ thesis, Ruprecht-Karls-Universität Heidelberg, 1998. 